\title{
ADF-STEM Imaging of Dopants and Defect Nanoclusters in Si
}

\author{
P. M. Voyles, ${ }^{*}$ D. A. Muller,** and J. L. Grazul**
}

*Dept. of Materials Science and Engineering, University of Wisconsin - Madison, 1509 University Ave., Madison, WI, 53706-1595; voyles@engr.wisc.edu **Bell Labs, Lucent Technologies, 700 Mountain Ave., Murray Hill, NJ 07974-0636

The ability to introduce free carriers into a silicon crystal by impurity doping has emerged as one of the potential roadblocks to the continued decrease in size of silicon microelectronic devices [1]. Future devices may require carrier (and thus dopant) concentrations $>10^{20} \mathrm{~cm}^{-3}$ in a volume who's smallest dimension is $<50 \mathrm{~nm}[2,3]$. Along with significant problems in device manufacturing, this produces (at least) two important challenges for microanalysis: (1) determining the impurity spatial distribution with sub-nm spatial resolution. (2) determining the structure of the defect responsible for the final limit on the free carrier concentration that can be achieved by doping.

Both of these challenges can be fruitfully addressed using annular dark-field (ADF) "Z-contrast" STEM imaging, provided we have a clear understanding of the physics of image formation. In the simplest picture, the ADF image intensity $I(\mathbf{r}) \propto P(\mathbf{r}) \otimes t(\mathbf{r})$, where $P(\mathbf{r})$ is the probe intensity and $t(\mathbf{r})$ is the specimen transmission function. This picture is inadequate for a zone-axis crystal because of very strong channeling of the incident probe causing it to change shape as a function of depth [4, 5]. A better picture is that the contribution to $I(\mathbf{r})$ from an atom at $\left(\mathbf{r}_{\mathbf{a}}, z_{a}\right)$ is proportional to the channeling-induced probe intensity at that atom $P\left(\mathbf{r}_{\mathbf{a}}, z_{a}\right)$ weighted by $t\left(\mathbf{r}_{\mathbf{a}}, z_{a}\right) \propto Z_{a}^{1.7}[6]$,

$$
\begin{aligned}
d I / d z(\mathbf{r}) & =P(\mathbf{r}, z) \otimes t(\mathbf{r}, z), \text { implying } \\
I(\mathbf{r}) & =\int_{0}^{t} P(\mathbf{r}, z) \otimes t(\mathbf{r}, z) d z .
\end{aligned}
$$

Fig. 1 shows plane-wave frozen-phonon multislice simulations indicating that $d I / d z$ and $P(\mathbf{r}, z)$ calculated for a probe on a $\langle 110\rangle \mathrm{Si}$ column are proportional, as Eq. (1) predicts.

The excess intensity from substitutional dopant atom $I_{d} / I_{S i}$ is therefore determined both by its $Z$ and channeled probe it sees, so $I_{d} / I_{S i}$ for a single impurity depends on the impurity depth in the sample. If the sample has low enough doping and small enough thickness, $\left(<7.7 \times 10^{21} \mathrm{~cm}^{-3}\right.$ at $50 \AA$ for $\langle 110\rangle$ $\mathrm{Si}$ at $200 \mathrm{kV}$ ), this could be used to determine the depth and therefore the full three-dimensional position of the impurities. If we average over a number of columns on the order of the sample thickness ( 10-100), the channeling contribution to Eq. (1) cancels out of $I_{d} / I_{S i}$, and it becomes

$$
\frac{I_{d}}{I_{S i}}-1=n_{d} \frac{\left(Z_{d}^{1.7}-Z_{S i}^{1.7}\right)}{Z_{S i}^{1.7}},
$$

where $n_{d}$ is the number fraction of dopant atoms [6]. For $d=\mathrm{Sb}$ and $I_{d} / I_{S i}=1 \%$, we can quantitatively measure dopant concentrations of $\sim 10^{19} \mathrm{~cm}^{-3}$ by averaging over 25 atomic columns.

Channeling can also help address the second challenge, determining the structure of individual defect clusters. Defects involving two or more dopant atoms and either a Si vacancy $[7,8]$ or a Si bond reconstruction [9] have been proposed. We have recently demonstrated ADF-STEM images showing contrast from individual $\mathrm{Sb}$ atoms in $\mathrm{Si}$ [10]. These images showed that the defect can contain only two dopant atoms. One structural difference between various pair defects is the 


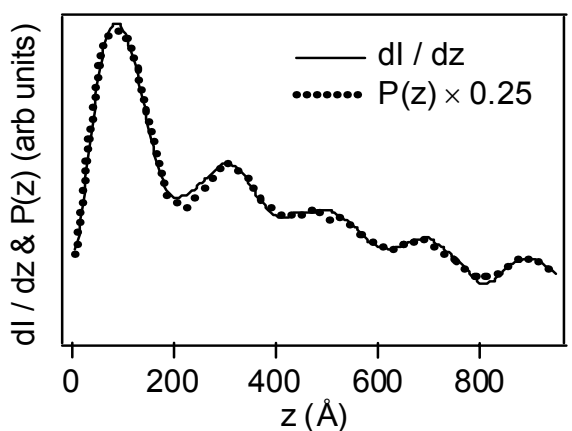

Fig. 1: $d I / d z$ and $P(z)$ simulated for a $200 \mathrm{kV} 1.0 \mathrm{~mm} \mathrm{C}_{\mathrm{s}}$ probe incident on an atomic column of $\langle 110\rangle \mathrm{Si}$. They are proportional, indicating that Eq. (1) is valid.

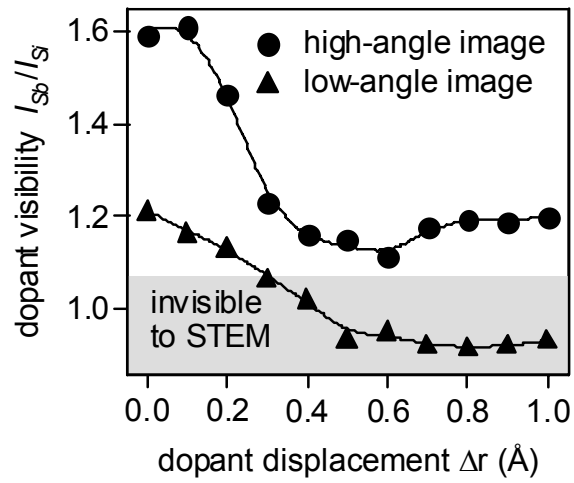

Fig. 2. Simulated $I_{d} / I_{S i}$ for $d=\mathrm{Sb}$ as a function of $\Delta r$.

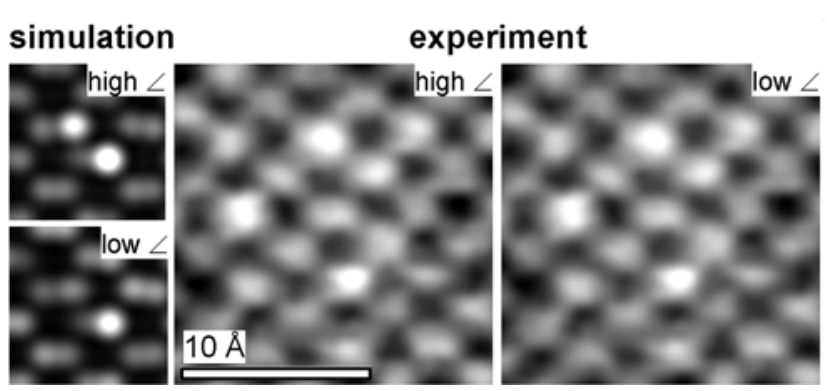

Fig 3: (left) Simulated images of a defect in which one dopants has a large $\Delta r=0.7 \AA$ and is invisible in the low-angle image. (right) Experimental images of Sb-doped $\mathrm{Si}$. The brightest columns contain one $\mathrm{Sb}$ atom, and are bright in both images, indicating $\Delta r$ for these $\mathrm{Sb}$ atoms is $<\sim 0.3 \AA$. Of several hundred $\mathrm{Sb}$ atoms in the full-field high-angle images, only a statistically insignificant fraction (similar to the false positive detection rate of $\sim 3 \%$ ) were not found in the low-angle images. distance $\Delta r$ that the dopants are pulled off their substitutional positions. Channeling draws the probe tightly onto the atomic column, so the $I_{d} / I_{S i}$ of dopants falls with increasing $\Delta r$, as shown in simulations in Fig. 2.

Conclusions based on absolute electron micrograph intensities are notoriously unreliable, so we use the Si lattice as an online standard. At lower detector angles, the Si lattice contrast grows stronger, reducing $I_{d} / I_{S i}$. Therefore, while essentially all of the dopants will appear in a high-angle image [10], dopants with large $\Delta r$ will not appear in a low-angle image of the same area. A STEM can acquire both images simultaneously, so they are guaranteed to be in exact registration.

Using this technique, we have determined that the primary deactivating defect in Sb-doped Si has $\Delta r<0.3 \AA$, which is inconsistent with either of the dominant defects in the literature [11]. Sample images are shown in Fig. 3. We have therefore proposed a new defect, in which most of the structural dislocation is centered on a Si Frenkel pair, leaving the $\mathrm{Sb}$ atoms relatively undisturbed [11].

[1] P. A. Packan, Science 285, 2079 (1999).

[2] H.-J. Gossmann, C. S. Rafferty, and P. Keys, Mat. Res. Soc. Symp. 610, B1.2.1 (2000).

[3] International Technology Roadmap for Semiconductors, Update 2000 (Semiconductor Industry Association, 2000).

[4] J. Fertig and H. Rose, Optik 59, 407 (1981).

[5] S. E. Hillyard and J. Silcox, Ultramicroscopy 52, 325 (1993).

[6] R. R. Vanfleet, et al., in Characterization and Metrology for ULSI Technology: 1998

International Conference, edited by D. G. Seiler, et al., 1998), p. 901.

[7] K. C. Pandey et al., Phys. Rev. Lett. 61, 1282 (1988).

[8] M. Ramamoorthy and S. T. Pantelides, Phys. Rev. Lett. 76, 4853 (1996).

[9] D. J. Chadi et al., Phys. Rev. Lett. 79, 4834

(1997).

[10] P. M. Voyles et al., Nature 416, 826 (2002).

[11] P. M. Voyles, et al., Phys. Rev. Lett.

(submitted). 